\title{
Long segment spinal neurofibroma: An unusual cause of low back pain
}

Sir,

Spinal neurofibromas are uncommon, accounting for $3 \%$ of all spinal tumors. ${ }^{[1]}$ Neurofibroma without other stigmata of neurofibromatosis has been reported in numerous locations, including the spine, retroperitoneal space, mandible, buccal mucosa, nose, bladder, and scrotum. ${ }^{[2]}$ We report a case of spinal neurofibroma involving long segment of spinal axis from D10 to S1 presenting with acute exacerbation of low back pain, feigning an acute disc prolapse. Spinal neurofibroma involving nine segments without other stigmata of neurofibromatosis is one of the rare cases reported in the literature. Low back pain should not be taken lightly and should be thoroughly investigated.

A 55 year-old lady presented with low back pain radiating to both lower limbs ( $\mathrm{L}>\mathrm{R}$ ), on and off for 10 years, with sudden increase in low back pain and weakness of both lower limbs for the past 15 days. There was no bladder/bowel dysfunction. On examination, tenderness was present over dorsolumbar spine, areflexia of lower limbs with power $\mathrm{R}-4 / 5$ and $\mathrm{L}-3 / 5$, bilateral EHL weakness, and paraesthesia below L1 dermatome. At this juncture, cauda equina syndrome was considered in view of asymmetrical weakness of lower limbs which could be due to an acute disc prolapse or an extruded disc.

Plain X-ray showed widening of spinal canal and scalloping of posterior margins of L3-S1 vertebrae, suggestive of a chronic lesion-like neurofibroma. Patient had no neurocutaneous markers and no family history of neurofibromatosis. MRI showed mildly enhancing intradural mass lesion from D10-S1 appearing iso/ hypointense on T1, hyperintense on $\mathrm{T} 2$, and extending into the paraspinal region through intervertebral foramina at L3-L4 level [Figure 1].

Per-operatively, tumor portrayed pebble stacker appearance from D10 up to the sacrum [Figure 2]. Tumor was adherent to spinal cord, nerve roots, and found compressing the cord. Subtotal resection of tumor was done, fearing worsening of the present neurological status.

Histopathology reported as features suggestive of neurofibroma.

Postoperatively, over a period of 20 days patient showed improvement in motor power, and was able to stand and walk with support.

To conclude, spinal neurofibroma, although uncommon, is an important consideration in the differential diagnosis of low back pain or radicular pain with neurological deficits. Early recognition facilitates timely treatment, potentially minimizes neurological morbidity, and may improve outcome. Resection of tumor should be the primary modality of treatment wherever feasible. Subtotal resection or decompressive surgery is favored when tumor is closely adherent to nerve roots or vital structures.
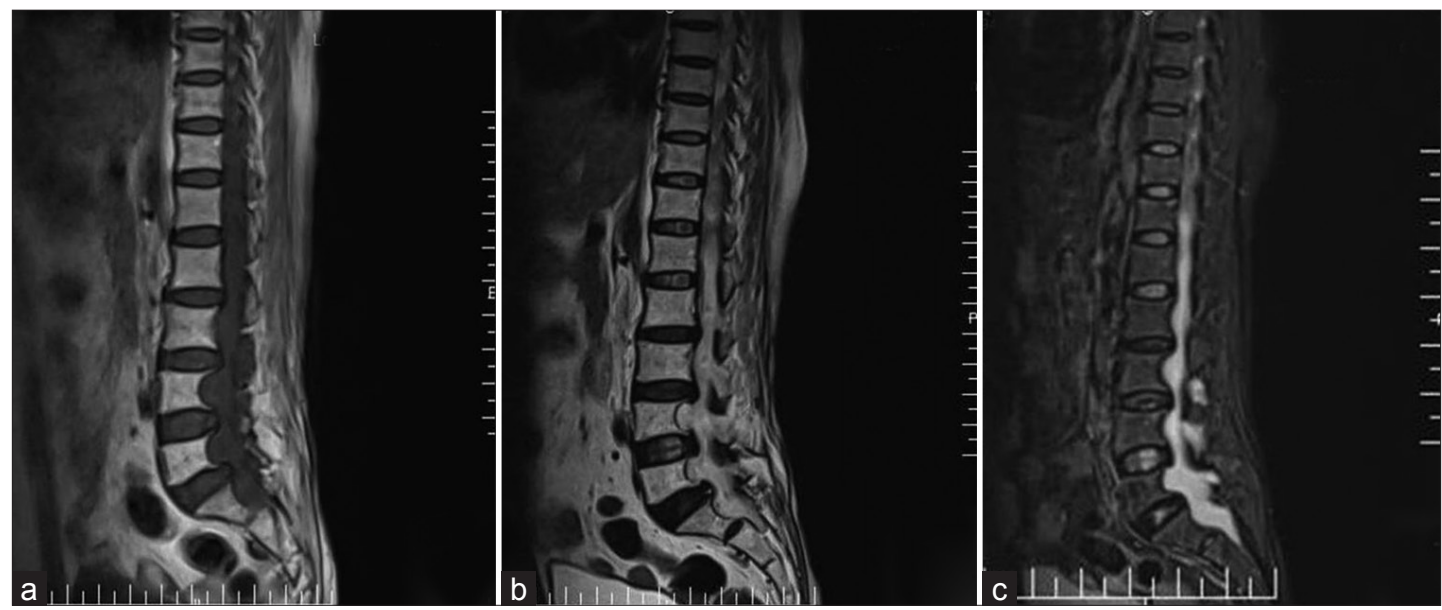

Figure 1: MRI dorsolumbar spine showing an irregular abnormal signal intensity lesion from D10-S1, (a) appearing hypointense on T1 and (b and c) hyperintense on T2 


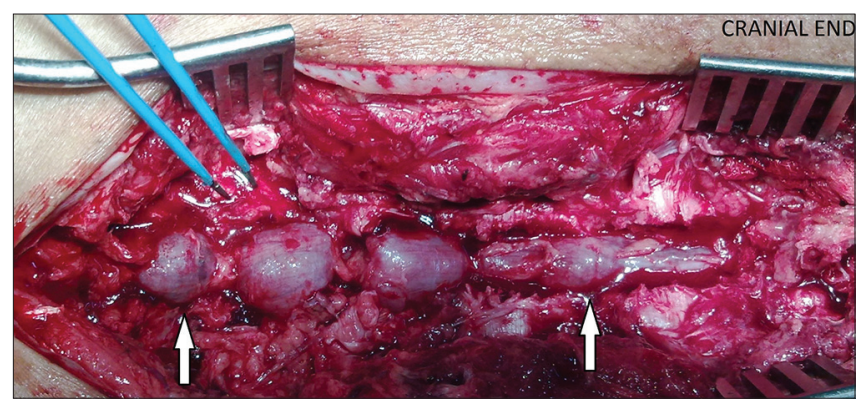

Figure 2: Per-operative "pebble stacker appearance" of intradural spinal tumor (arrows pointing to the tumor)

N. Chandrashekar, Karthik K. Prasad ${ }^{1}$

Departments of Neurosurgery and ${ }^{1}$ Surgery, Mysore Medical College and Research Institute, K R Hospital, Mysore, Karnataka, India. E-mail: drkrishkarthik@gmail.com

\section{REFERENCES}

1. Seppälä MT, Haltia MJ, Sankila RJ, Jääskeläinen JE, Heiskanen O. Long-term outcome after removal of spinal neurofibroma. J Neurosurg 1995;82:572-7.

2. Yang L, Robertson T, Tollesson G, Francis L, Campbell D, Winter C. An unusual presentation of a solitary benign giant neurofibroma case report. J Neurosurg Spine 2009;11:49-52.

\begin{tabular}{|l|l|}
\hline \multicolumn{2}{|c|}{ Access this article online } \\
\hline Quick Response Code: & Website: \\
\hline & www.ijns.in \\
\cline { 2 - 2 } & \\
\hline
\end{tabular}

